\title{
AB-INITIO STUDY OF SURFACE ENERGIES AND STRUCTURAL INFLUECE OF VACANCIES IN TITANIUM NITRIDE NANOLAYER
}

\author{
1,2Miroslav LEBEDA, ${ }^{1}$ Petr VLČÁK, ${ }^{2}$ Petr VEŘTÁT, ${ }^{3} J a n$ DRAHOKOUPIL \\ ${ }^{1}$ Department of Physics, Faculty of Mechanical Engineering, Czech Technical University in Prague, \\ lebedmi2@fjfi.cvut.cz,petr.vlcak@fs.cvut.cz \\ ${ }^{2}$ Department of Solid State Engineering, Faculty of Nuclear Sciences and Physical Engineering, \\ Czech Technical University in Prague, vertapet@ffifi.cvut.cz \\ ${ }^{3}$ Department of Material Analysis, Institute of Physics, Czech Academy of Sciences, draho@fzu.cz
}

https://doi.org/10.37904/nanocon.2020.3706

\begin{abstract}
Titanium nitride $(\mathrm{TiN})$ is largely applied as protective nanolayer of medical titanium implants due to its significant positive influence on the surface properties such as friction and corrosion resistance. During the process of its phase formation, occurrence of the point defects is probable and can potentially affect various material properties, e.g. change the lattice parameters. The surface energies of 8 crystallographic planes and effects of nitrogen vacancies on the lattice parameter in rock salt-like structure of TiN $(\delta-\mathrm{TiN})$ were studied using ab-initio method of density functional theory (DFT) with the generalized gradient approximation functional (GGA) as parametrized by Perdew, Burke and Ernzerhof (PBE). TiN supercell with 64 atomic positions was used for the calculation of defects influence and the results are thoroughly discussed in the context of available theoretical and experimental literature data. The linear decrease of lattice parameter with the increasing presence of nitrogen vacancies up to ca. $80 \%$ was observed. The surface energy of (100) crystallographic plane is two times lower $\left(1.499 \mathrm{~J} / \mathrm{m}^{2}\right)$ than for the rest of the studied planes.
\end{abstract}

Keywords: Titanium nitride, $\mathrm{TiN}_{\mathrm{x}}$, ab initio simulation, density functional theory, DFT, vacancies, lattice parameter, surface energy

\section{INTRODUCTION}

Titanium and its alloys are nowadays widely used as implant materials in biomedical industry due to their generally great mechanical, physical and chemical properties. By various types of surface treatment, their surface properties (such as friction or corrosion resistance) are often suitably modified without changing the required bulk ones (such as internal bulk modulus) [1]. One of the most prominent and controllable surface modification methods is the implantation of charged particles (e.g. ions of nitrogen, hydrogen, oxygen, potassium, carbon, argon, silver) under the top surface layer intervening depth of the sample typically about $100 \mathrm{~nm}$ [2]. Changes of material properties are primarily caused by the formation of point defects, dislocations and potential new phases in the affected area, all of which are directly related to the depth concentration profiles of implanted ions and their dose [3]. In case of $\mathrm{N}$ ion implantation into the $\alpha$-Ti matrix ( $\left.\mathrm{P}_{3} / \mathrm{mmc}, \# 194\right)$ [4], titanium nitride (TiN) with rock salt-like structure (ס-TiN, $F m \overline{3} m$, \#225) is primarily formed [5].

Metallic TiN has wide range of interesting properties such as high melting point, ultra-hardness, excellent mechanical resistance or good electric and thermal conductivity [6]. Therefore, it is being largely applied as coating nanolayer. During the modification of $\alpha$-Ti with $\mathrm{N}$ ion implantation, the occurrence of high-density of $\mathrm{N}$ and Ti vacancies, substitutions or interstitials can significantly modify physical properties in the implanted zone, e.g. change the lattice parameters. Thus, knowledge of point defects and their influence is essential for understanding the behavior of our material. Particularly other important characteristics are the surface energies 
as they play significant role in epitaxial film growth of TiN or in the explanations of particle (e.g. hydrogen, oxygen) absorptions on its given surface layer [7].

It is often desirable to have theoretical predictions of all the mentioned characteristics and effects as they can provide a meaningful insight to the material behaviour or the processes occurring during its modifications. Development of ab-initio simulation methods, such as density functional theory (DFT), allowed performing aforementioned predictions and had become broadly used in the last decades. In this study, we provide DFT predictions of the mean effects of vacancies on the lattice parameter in rock salt-like structure of TiN. We also present the calculations of surfaces energies of TiN for 8 crystallographic planes and compare all our results with published studies for the cases where data was available.

\section{COMPUTATIONAL DETAILS}

DFT simulations were performed within the CASTEP (Cambridge Serial Total Energy Package) code [8]. The exchange-correlation energy functional was approximated by means of the GGA in PBE parametrization [9]. Iterative solutions of Kohn-Sham equations are derived with plane-wave basis for linear expansion of the electronic wavefunctions. Suitable value of energy cutoff $E_{\text {cut }}$, determining the number of plane waves used in the expansion, was found by the following algorithm: The total free energy of the fcc-TiN unit cell (with 8 atoms) was calculated with predefined ultra-fine settings while changing only the values of $E_{\text {cut }}$ ranging from 140 to $600 \mathrm{eV}$ with the step of $20 \mathrm{eV}$ (Figure 1, Left, red marks). We set the energy cut-off at $360 \mathrm{eV}$ to achieve the calculation convergence of at least $0.02 \mathrm{eV}$ per atom with respect to this parameter. Similar convergence algorithm was used to find the suitable parameters of the Monkhorst-Pack k-point mesh [10] for the sampling of Brillouin zone. We selected $8 \times 8 \times 8$ (Figure 1, Left, blue marks) as the energy convergence per atom with respect to the k-point sampling is here lesser than $0.01 \mathrm{eV}$. To further reduce the number of plane wave coefficients, we used the ultrasoft pseudopotential of the Vanderbilt type [11]. The system was considered be fully relaxed when the magnitude of the forces acting on all atoms was smaller than $0.01 \mathrm{eV} / \AA$ and the difference of energy per atom between consecutive iterates has decreased under $5 \cdot 10^{-6} \mathrm{eV} / \mathrm{atom}$.
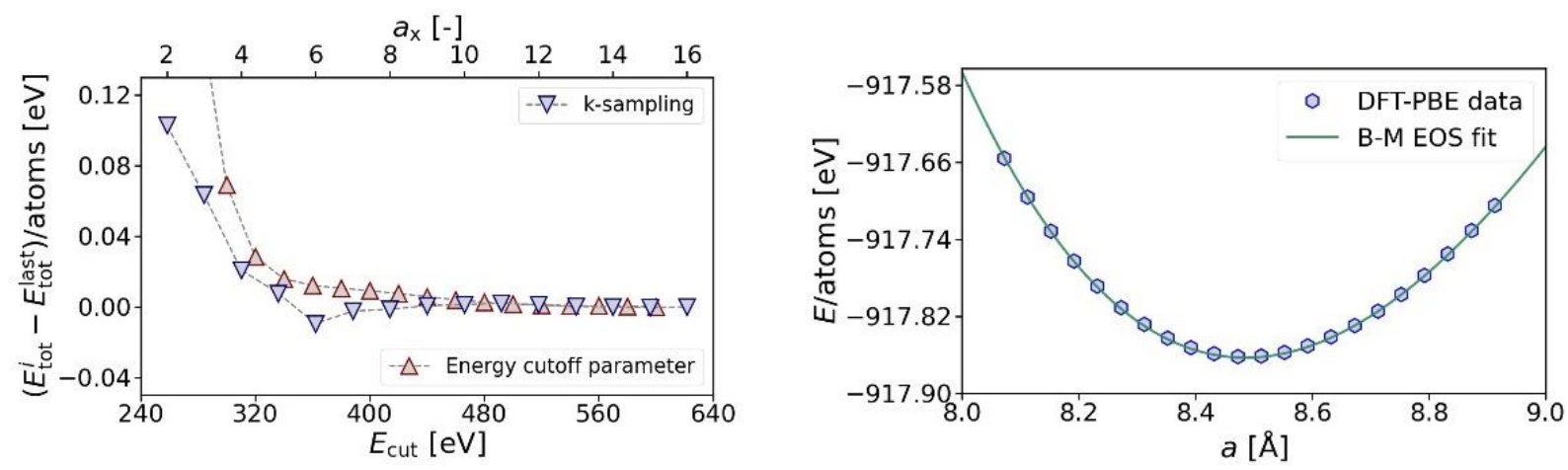

Figure 1 Left: Convergence of the fcc TiN total energy per atom with respect to the cutoff energy parameter $E_{\text {cut }}$ and $a_{\mathrm{x}} \times a_{\mathrm{x}} \times a_{\mathrm{x}}$ k-points from the Monkhorst-Pack grid. Right: Quality of DFT data fit with Birch-Murnaghan equation of state.

For the surface energies calculations, we have used a grid of $8 \times 8 \times 1$, where $\times 1$ is the usual choice in the direction perpendicular to the surface. This accounts the fact that the length of the supercell along this direction includes a vacuum region. Calculations of vacancies were performed on the TiN supercell containing 64 atomic sites. Monkhorst-Pack grid was reduced to $4 \times 4 \times 4$ as the lattice parameters are now twice the bigger the unit cell containing 8 atoms. This provides a set of k-points with equal density in each axis direction of the reciprocal space. The placement of defects for their given number was randomized using a custom script in PERL language. Being interested only in mean effects of vacancies on lattice parameters, we kept the supercells as cubic and calculated their dependences of total energy per atom on the lattice parameter $a$. 
The range of changes of $a$ was within $10 \%$ of the computed equilibrium value of non-defected TiN $(8.492 / 2=$ $4.246 \AA$ ) with the step of $0.04 \AA$. The obtained dependences were fitted by the Birch-Murnaghan equation of states (EOS) [12]:

$$
E(a)=E_{\mathrm{c}}+\frac{9 a_{\mathrm{e}} B}{16}\left\{\left[\left(\frac{a_{\mathrm{e}}}{a}\right)^{\frac{2}{3}}-1\right]^{3} B^{\prime}+\left[\left(\frac{a_{\mathrm{e}}}{a}\right)^{\frac{2}{3}}-1\right]^{2}\left[6-4\left(\frac{a_{\mathrm{e}}}{a}\right)^{\frac{2}{3}}\right]\right\}
$$

where fitting parameters have the following physical meaning: $E_{\mathrm{c}}$ is the cohesive energy, $a_{\mathrm{e}}$ the equilibrium lattice parameter, $B$ the bulk modulus and $B^{\prime}$ its derivation. Quality of this fit is illustrated for the one case in Figure 1, Right.

\section{RESULTS AND DISCUSSION}

\subsection{Calculations of TiN surface energies}

We have studied the TiN surface energies $E_{\mathrm{DFT}}^{\text {Surf }}$ of (100), (110), (111), (211), (221), (311), (321) and (322) cleavage planes. The bulk energy per atom was set as the energy of geometrically optimized TiN structure. Resulting lattice parameter $a=4.246 \AA$ was in very good agreement with the experimental value $4.238 \AA$ [13] (relative deviation of $0.2 \%$ ). After that, all the crystallographic planes were cleaved from this optimized structure. Neither the symmetric nor the asymmetric surface relaxations were allowed as well as the surface reconstructions. In all the considered planes (except for (111) and (311)), we have used the smallest possible surface cell for all slab calculations and tested the effect of different numbers of atomic layers ranging from 4 to 12 (with 8 to 24 atoms) on the surface energy. For (111) and (311), we had to choose non-primitive surface cell in order to keep the consistence of the same number of atoms in layers with the rest of the studied planes. Since (111) and (311) surfaces have single species termination in rock salt-like structure, there are three possibilities of cleavage. Both sides are either Ti or $\mathrm{N}$ terminated, or one is terminated with Ti layer while the other one with $\mathrm{N}$. We have chosen the later, mixed state. This should lead to an average value of surface energy between the two different terminations with the same atomic species [3].

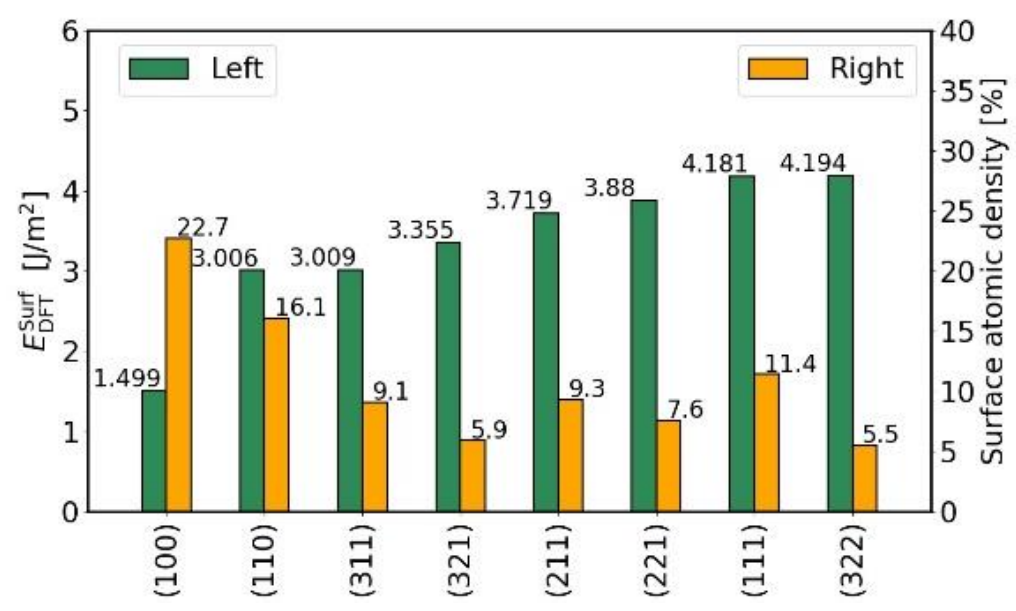

Figure 2 Descending order from the most stable surface to the least stable one in TiN. Given data are calculated by means of the GGA-PBE functional with 12 atomic layers (24 atoms in the slab) and $10 \AA$ thickness of vacuum. For each plane, corresponding atomic surface density is also shown.

Resulted surface energies are summarized in Table 1. Our values are well converged with respect to the numbers of atomic layers in the slab as the surface energy changes are not greater than $0.1 \mathrm{~J} / \mathrm{m}^{2}$ from 8 layers (16 atoms). Descending order from the most stable surface to the lowest stable one is shown in Figure 2 with 
the corresponding atomic surface densities (energies are in units $\mathrm{J} / \mathrm{m}^{2}=16.022 \mathrm{eV} / \AA^{2}$ and for the vacuum thickness of $10 \AA$ with 24 atoms in the slab). Typically, the most stable surfaces are the ones with highest surface densities. This agrees for the $(100)\left(1.499 \mathrm{~J} / \mathrm{m}^{2}\right)$ as it has significantly higher density $(22.7 \%)$ than the second most stable (110) $\left(3.006 \mathrm{~J} / \mathrm{m}^{2}\right)$ surface with $16.1 \%$.

Table 1 DFT GGA-PBE calculations of TiN surface energies. Vacuum thickness was set to $10 \AA$. Surface relaxations and reconstructions are not considered. For the (111) and (311), one side was terminated with $\mathrm{N}$ atomic plane while the other with $\mathrm{Ti}$. Comparison (percentage of the relative deviation) with the closest reference value $[3,7]$ is given for the cleavage planes where data was found.

\begin{tabular}{|c|c|c|c|c|c|c|c|c|}
\hline $\begin{array}{l}\mathrm{N} . \text { of } \\
\text { layers } \\
\text { (atoms) }\end{array}$ & $\begin{array}{c}E_{\mathrm{PBE}}^{(100)} \\
\left.\mathrm{J} / \mathbf{m}^{2}\right)\end{array}$ & $\begin{array}{c}E_{\mathrm{PBE}}^{(110)} \\
\left(\mathbf{J} / \mathbf{m}^{2}\right)\end{array}$ & $\begin{array}{c}E_{\mathrm{PBE}}^{(\mathbf{1 1 1})} \\
\left(\mathrm{J} / \mathbf{m}^{2}\right) \\
\text { Ti-N term. }\end{array}$ & $\begin{array}{l}E_{\mathrm{PBE}}^{(211)} \\
\left(\mathrm{J} / \mathrm{m}^{2}\right)\end{array}$ & $\begin{array}{c}E_{\mathrm{PBE}}^{(221)} \\
\left(\mathrm{J} / \mathrm{m}^{2}\right)\end{array}$ & $\begin{array}{c}E_{\mathrm{PBE}}^{(311)} \\
\left(\mathrm{J} / \mathbf{m}^{2}\right) \\
\mathrm{Ti}-\mathrm{N} \text { term. }\end{array}$ & $\begin{array}{c}E_{\mathrm{PBE}}^{(321)} \\
\left(\mathrm{J} / \mathrm{m}^{2}\right)\end{array}$ & $\begin{array}{c}E_{\mathrm{PBE}}^{(322)} \\
\left(\mathrm{J} / \mathrm{m}^{2}\right)\end{array}$ \\
\hline $4(8)$ & $\begin{array}{c}1.580 \\
(+3.3 \%)\end{array}$ & $\begin{array}{c}3.013 \\
(+5.0 \%)\end{array}$ & $\begin{array}{c}4.420 \\
(+5.3 \%)\end{array}$ & 3.925 & 3.977 & 3.151 & 3.538 & 4.519 \\
\hline $6(12)$ & $\begin{array}{c}1.525 \\
(-0.3 \%)\end{array}$ & $\begin{array}{c}3.011 \\
(+4.9 \%)\end{array}$ & $\begin{array}{c}4.163 \\
(-0.8 \%)\end{array}$ & 3.782 & 4.072 & 3.098 & 3.387 & 4.341 \\
\hline $8(16)$ & $\begin{array}{c}1.529 \\
(-0.1 \%)\end{array}$ & $\begin{array}{c}3.030 \\
(+5.6 \%)\end{array}$ & $\begin{array}{c}4.206 \\
(+0.2 \%)\end{array}$ & 3.730 & 3.926 & 3.013 & 3.313 & 4.382 \\
\hline $10(20)$ & $\begin{array}{c}1.497 \\
(-2.2 \%)\end{array}$ & $\begin{array}{c}3.011 \\
(+4.9 \%)\end{array}$ & $\begin{array}{c}4.176 \\
(-0.5 \%)\end{array}$ & 3.736 & 3.896 & 3.002 & 3.365 & 4.277 \\
\hline $12(24)$ & $\begin{array}{c}1.499 \\
(-2.0 \%) \\
\end{array}$ & $\begin{array}{c}3.006 \\
(+4.7 \%)\end{array}$ & $\begin{array}{c}4.181 \\
(-0.4 \%) \\
\end{array}$ & 3.719 & 3.880 & 3.009 & 3.355 & 4.194 \\
\hline Refer. & $\begin{array}{c}1.530[3], \\
\mathrm{rPBE} \\
1.602[7], \\
\mathrm{PW} 91\end{array}$ & $\begin{array}{c}2.870[3], \\
\operatorname{rPBE}\end{array}$ & $\begin{array}{c}4.198[7], \\
\text { PW91 } \\
\begin{array}{c}5.080[3], \\
\text { rPBE }\end{array}\end{array}$ & - & - & - & - & - \\
\hline
\end{tabular}

\subsection{Nitrogen vacancies in $\mathrm{TiN}$ and their influence on lattice parameter}

We present DFT prediction of the mean effects of nitrogen vacancies on the lattice parameter $a$ in fcc TiN $\mathrm{N}_{1-x}$. To minimize the interactions between vacancies themself for periodic boundary calculations, the TiN supercell with 64 atomic sites was chosen. Within this supercell, vacancies had been created ranging from $3.13 \%$ to $100.00 \%$ with the step of $3.13 \%$ up to the $12.50 \%$ and from this point, with the step of $6.25 \%$ (corresponding to creation of two vacancies from initial $32 \mathrm{~N}$ atoms). Resulted dependence of the lattice parameter $a$ on $\mathrm{N}$ vacancies concentration (as $100 \times x$ in $\mathrm{TiN}_{1-x}$ ) from the least-square best-fit with Birch-Murnaghan EOS is presented in Figure 3. We observed linear decrease in $a$ with possible linear regression $a=-0.00009261 x+$ 4.247 up to the $80 \%$ of vacancy concentration $x$ (up to TiN 0.2 stoichiometry). The stability of $\delta$-TiN $\mathrm{N}_{1-x}$ phase was experimentally observed up to $63 \%$ of $N$ vacancy concentration [14]. Our calculation shows that the cubic phase of $\mathrm{TiN}_{1-x}$ could be stable/metastable with even higher concentration of $\mathrm{N}$ vacancies, up to ca. $80 \%$, as the slope of the lattice parameter starting to decrease from this point in much steeper way.

In [15,16], similar calculations were performed on smaller supercell consisting of 8 atomic positions of fcc TiN using the Full Potential-Linear Muffin Tin Orbital (FP-LMTO) implementation of DFT with local density approximation (LDA). However, there arising direct disadvantages using this type of cell as the number of vacancy concentrations is strictly limited $(0 \%, 25 \%, 50 \%, 75 \%$ and $100 \%)$, and the self-interactions of defects within periodic boundary conditions are less eliminated. Very recently, such simulations were extended in [17] using GGA-PBE functional on the supercell with the same size as ours (64 atomic positions). We used all these theoretical reference data as well as available experimental results [17-20] and have compared them with linear regression of own calculated lattice parameter dependence in Table 3. Our results are for 0.00 , $1.00,20.00,49.00$ and $50.00 \%$ of $\mathrm{N}$ vacancy concentration in very good agreement with experimentally 
derived lattice parameters, as the maximum relative deviation was $0.61 \%$ for $\mathrm{TiN}_{0.80}$ [19]. Comparing to the theoretical prediction [17], their data starting at very similar value $(0.00 \%$ deviation at $x=3.13 \%)$ and slightly differs at $x=25.00 \%$ with $-0.26 \%$ error from our results. Overall, with the highest relative deviation of $2.14 \%$ for $\mathrm{TiN}_{0.25}$ [16], there is no discrepancy between data in literature and our calculated prediction.

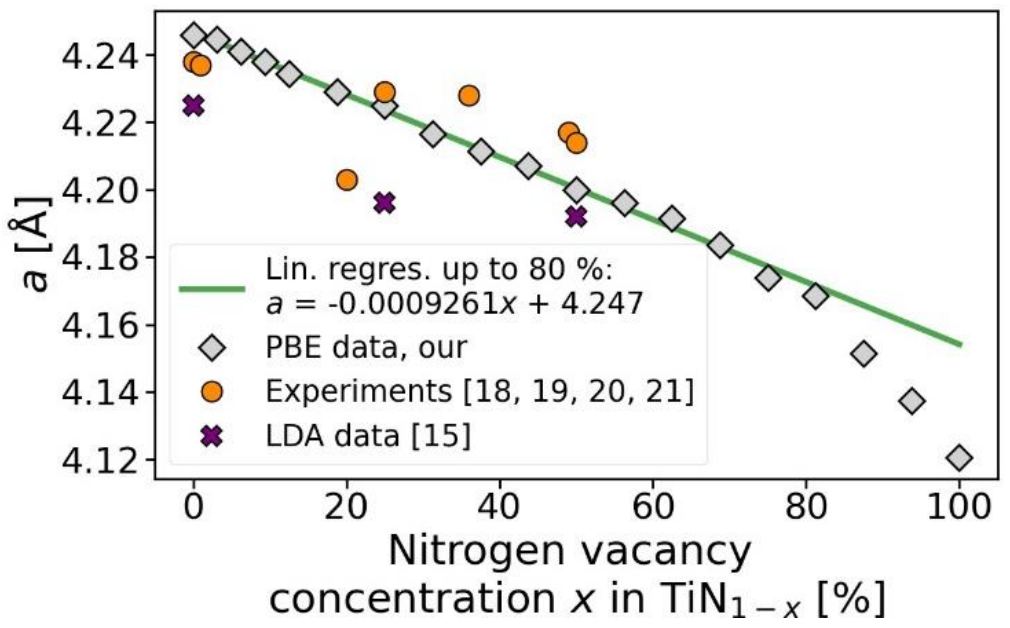

Figure 3 DFT GGA-PBE calculations of $\mathrm{TiN}_{1-x}$ lattice parameter changes with increasement presence of nitrogen vacancies $(100 \times x)$ performed on the supercell with 64 atomic position sites.

Table 3 Comparison of our GGA-PBE calculations of TiN $\mathrm{N}_{1-x}$ lattice parameter dependence on $\mathrm{N}$ vacancy concentration with theoretical and experimental data from available literature

\begin{tabular}{|c|c|c|c|}
\hline $\begin{array}{l}x \text { in } \mathrm{TiN}_{1-x} \\
(\times 100 \%)\end{array}$ & $\begin{array}{c}a_{\text {ref }}, \text { references } \\
(\AA)\end{array}$ & $\begin{array}{c}a_{\text {our }}, \text { our PBE } \\
\text { results, linear fit }(\AA)\end{array}$ & $\frac{a_{\text {our }}-a_{\text {ref }}}{a_{\text {ref }}}(\%)$ \\
\hline 0.00 & 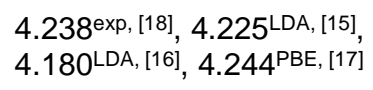 & 4.247 & $\begin{array}{l}+0.21,+0.52 \\
+1.60,+0.07\end{array}$ \\
\hline 1.00 & $4.237 \exp ,[19]$ & 4.246 & +0.21 \\
\hline 3.13 & $4.244^{\mathrm{PBE},[17]}$ & 4.244 & 0.00 \\
\hline 6.26 & $4.243^{\mathrm{PBE},[17]}$ & 4.241 & -0.04 \\
\hline 12.50 & $4.242^{\mathrm{PBE},[17]}$ & 4.235 & -0.16 \\
\hline 20.00 & $4.203^{\exp ,[19]}$ & 4.228 & +0.61 \\
\hline 25.00 & 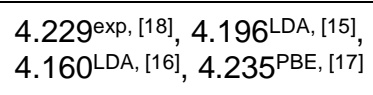 & 4.224 & $\begin{array}{l}-0.12,+0.66 \\
+1.53,-0.26\end{array}$ \\
\hline 36.00 & $4.228 \exp ,[21]$ & 4.214 & -0.34 \\
\hline 49.00 & $4.217^{\exp ,[20]}$ & 4.202 & -0.36 \\
\hline 50.00 & 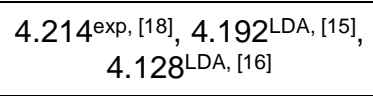 & 4.201 & $\begin{array}{l}-0.32,+0.21 \\
+1.76\end{array}$ \\
\hline 75.00 & $4.090^{\text {LDA, }[16]}$ & 4.178 & +2.14 \\
\hline
\end{tabular}

\section{CONCLUSION}

We have determined surface energies of 8 crystallographic planes in TiN by means of the ab-initio simulation method of DFT with GGA-PBE exchange-correlation functional. The most stable is (100) which agrees with the fact it has significantly higher atomic surface density than the rest of the studied surface planes. Comparing with available published data for (100), (110) and (111) surfaces, our calculations are in very well agreement. We also used DFT GGA-PBE method to calculate influence of nitrogen vacancies on the lattice parameter $a$ 
in rock salt-like structure of $\mathrm{TiN}_{1-x}$. Linear decrease of $a$ with increasement in $\mathrm{N}$ vacancy concentration is observed and the linear regression of those data is possible up to $80 \%$ of vacancies presence. From this point, the slope of the lattice parameter is much steeper and one can predict that the metastability of cubic $\mathrm{TiN}_{1-x}$ phase will be close to $\mathrm{TiN}_{0.20}$ composition. To conclude, we have reached excellent correspondence with theoretical and experimental published literature data and extended calculated lattice parameter dependence of $\delta-\mathrm{TiN}_{1-x}$ on the full range of nitrogen vacancies with very good linear fit regression.

\section{ACKNOWLEDGEMENTS}

This work was supported by the Ministry of Health of the Czech Republic [project number NV19-08-00070] and the Grant Agency of the Czech Technical University in Prague [grant No. SGS19/190/OHK4/3T/14] and by the Operational Program Research, Development and Education financed by European Structural and Investment Funds and the Czech Ministry of Education, Youth and Sports [Project No. SOLID21 - CZ.02.1.01/0.0/0.0/16_019/0000760].

\section{REFERENCES}

[1] VLCAK, P. et al. Influence of surface pre-treatment with mechanical polishing, chemical, electrochemical and ion sputter etching on the surface properties, corrosion resistance and MG-63 cell colonization of commercially pure titanium. Mater. Sci. Eng. C. 2020, p. 111065.

[2] VLCAK, P. et al. Structural characterization and mechanical properties of a titanium nitride-based nanolayer prepared by nitrogen ion implantation on a titanium alloy. J. Nanomater. 2016, vol. 2016.

[3] MARLO, M., MILMAN, V. Density-functional study of bulk and surface properties of titanium nitride using different exchange-correlation functionals. Phys. Rev. B - Condens. Matter Mater. Phys. 2000, vol. 62, no. 4, pp. 28992907.

[4] VOHRA, Y. K., SPENCER, P. T. Novel y-phase of titanium metal at megabar pressures. Phys. Rev. Lett. 2001, vol. 86, no. 14, pp. 3068-3071.

[5] BOLOKANG, A. S., PHASHA, M. J. Formation of titanium nitride produced from nanocrystalline titanium powder under nitrogen atmosphere. Int. J. Refract. Met. Hard Mater. 2010, vol. 28, no. 5, pp. 610-615.

[6] BÈS, R. et al. First-principles study of rare gas incorporation in titanium nitride. Phys. Rev. B - Condens. Matter Mater. Phys. 2013, vol. 87, no. 2, pp. 1-10.

[7] GALL, D. et al. Pathways of atomistic processes on $\mathrm{TiN}(001)$ and (111) surfaces during film growth: An ab initio study. J. Appl. Phys. 2003, vol. 93, no. 11, pp. 9086-9094.

[8] CLARK, S. J. et al. First principles methods using CASTEP. Zeitschrift für Krist. Mater. 2005, vol. 220, no. 5-6, pp. 567-570.

[9] PERDEW, J. P. et al. Generalized gradient approximation made simple. Phys. Rev. Lett. 1996, vol. 77, no. 18, p. 3865.

[10] MONKHORST, H. J., PACK, J. D. Special points for Brillouin-zone integrations. Phys. Rev. B. 1976, vol. 13, no. 12 , p. 5188.

[11] VANDERBILT, D. Soft self-consistent pseudopotentials in a generalized eigenvalue formalism. Phys. Rev. $B$ 1990, vol. 41, no. 11, p. 7892.

[12] BIRCH, F. Finite elastic strain of cubic crystals. Phys. Rev. 1947, vol. 71, no. 11, p. 809.

[13] KOSOLAPOVA, T. Y. Handbook of high temperature compounds: properties, production, applications. CRC Press, 1990.

[14] PALTY, A. E. et al. Titanium-nitrogen and titanium-boron systems. New York University, 1954.

[15] GUEMMAZ, M. et al. Theoretical and experimental investigations on elastic properties of substoichiometric titanium nitrides: Influence of lattice vacancies. Int. J. Inorg. Mater. 2001, vol. 3, no. 8, pp. 1319-1321.

[16] DRIDI, Z. et al. First-principles calculations of vacancy effects on structural and electronic properties of TiCx and TiNx. J. Phys. Condens. Matter 2002, vol. 14, no. 43, pp. 10237-10249. 
[17] CATELLANI, A. et al. Tailoring the plasmonic properties of metals: The case of substoichiometric titanium nitride. Phys. Rev. Mater. 2020, vol. 4, no. 1, p. 15201.

[18] WRIEDT, H. A., MURRAY, J.L. The N-Ti (nitrogen-titanium) system. Bull. Alloy Phase Diagrams. 1987, vol. 8, no. 4, pp. 378-388.

[19] HÖCHST, H. et al. Photoemission study of the electronic structure of stoichiometric and substoichiometric TiN and ZrN. Phys. Rev. B. 1982, vol. 25, no. 12, pp. 7183-7191.

[20] GUEMMAZ, M. et al. Ion implantation processing of sub-stoichiometric titanium nitrides and carbonitrides: Chemical structural and micromechanical investigations. Appl. Phys. A Mater. Sci. Process. 1997, vol. 64, no. 4, pp. 407-415.

[21] JIANG, C.-C. et al. Non-stoichiometry of titanium nitride plates prepared by chemical vapour deposition. J. Alloys Compd. 1993, vol. 190, no. 2, pp. 197-200. 